\title{
ORBITS OF HIGHER-DIMENSIONAL HEREDITARILY INDECOMPOSABLE CONTINUA ${ }^{1}$
}

\author{
JAMES T. ROGERS, JR. ${ }^{2}$
}

\begin{abstract}
Let $X$ be a continuum. The following theorems are proved.
THEOREM. If $\operatorname{dim} X>1$, then $X$ contains uncountably many nonhomeomorphic continua.

THEOREM. If $\operatorname{dim} X>1$ and $X$ is hereditarily indecomposable, then $X$ has uncountably many orbits under the action of its homeomorphism group.
\end{abstract}

In 1970, Howard Cook [3] gave a beautiful proof that hereditarily equivalent continua are tree-like. In 1982, the author [9] proved that homogeneous, hereditarily indecomposable continua are tree-like. A step in both these proofs was to show that such continua must be one-dimensional.

In this paper, we give a simple and independent proof that these continua must be one-dimensional. Moreover, the results are even stronger: namely, if $X$ is a continuum and $\operatorname{dim} X>1$, then $X$ contains uncountably many nonhomeomorphic subcontinua. If, in addition, $X$ is hereditarily indecomposable, then $X$ has uncountably many orbits under the action of its homeomorphism group.

A continuum is a compact, connected, nonvoid metric space. A continuum is hereditarily equivalent if it is homeomorphic to each of its proper nondegenerate subcontinua. A continuum is indecomposable if it is not the union of two of its proper subcontinua. A continuum is hereditarily indecomposable if each of its subcontinua is indecomposable.

R. H. Bing [2] constructed examples of hereditarily indecomposable continua of dimension $n, 1 \leq n \leq \infty$. Bing [2] showed that, for $1<n<\infty$, no such continuum is homogeneous.

1. Old results from Poland. The joy and elegance of this paper is the way it pivots on two well-known results proved by Poles in the 1930s. The first result is the following theorem of Mazurkiewicz [7].

THEOREM 1. If $X$ is a continuum and $\operatorname{dim} x>1$, then there exists a weakly confluent map $f: X \rightarrow I^{2}$ of $X$ onto the unit square.

Recall that a map $f: Y \rightarrow Z$ between continua is weakly confluent if, for each subcontinuum $B$ of $Z$, there exists a subcontinuum $A$ of $Y$ such that $f(A)=B$.

Received by the editors October 8,1984 .

1980 Mathematics Subject Classification. Primary 54F20; Secondary 54F45, 54F50.

Key words and phrases. Continuum, homogeneous, aposyndetic, hereditarily equivalent, hereditarily indecomposable, dimension.

${ }^{1}$ This research was partially supported by NSF grant number MCS-8300569.

${ }^{2}$ Some of the ideas of this paper occurred to the author during the Semester on Topology at the Stefan Banach Mathematical Institute. The author thanks the National Academy of Sciences of the U.S. for its support and the Banach Institute for its hospitality and support. 
The second result concerns the Waraskiewicz spirals. Let $M$ denote the set of points in the plane with polar coordinates $(1, \theta)$, or $\left(1+e^{-\theta}, \theta\right)$ for $\theta \geq 0$, or $\left(1+e^{-\theta},-\theta\right)$ for $\theta \geq 0$. Thus $M$ is the union of $S^{1}$, a ray $R^{+}$spiralling in a counterclockwise direction onto $S^{1}$, and a ray $R^{-}$spiralling in a clockwise direction onto $S^{1}$.

The Waraskiewicz spirals are subcontinua of $M$. Each Waraskiewicz spiral is homeomorphic to a compactification of $[0,1)$ with remainder a circle. To determine a Waraskiewicz spiral, begin at the point with Cartesian coordinates $(2,0)$ and follow either $R^{+}$or $R^{-}$. At any time that the positive $x$-axis is crossed, feel free to change rays. This procedure determines the uncountable collection of continua called the Waraskiewicz spirals.

The importance of the Waraskiewicz spirals lie in the following fact: Waraskiewicz [11] proved that no continuum can be mapped onto all the Waraskiewicz spirals. R. Russo [10] has improved this proof; another proof appeared in $[6]$.

It is trivial to strengthen this theorem as follows:

THEOREM 2. Given any countable collection of continua, there exists a Waraskiewicz spiral $W$ such that no member of this collection can be mapped onto $W$.

The following conclusion is immediate from Theorems 1 and 2.

THEOREM 3. If $X$ is a continuum and $\operatorname{dim} X>1$, then $X$ contains uncountably many nonhomeomorphic subcontinua. In particular, hereditarily equivalent continua are one-dimensional.

The theorem also holds for those one-dimensional continua that admit a weakly confluent map onto a higher-dimensional continuum.

Anderson and Choquet [1] have used an inverse limit technique to construct a plane continuum with interesting restrictions on the existence of mappings between the subcontinua of this continuum. By replacing the rays spiralling onto $n$-ods in their construction with a Waraskiewicz spiral, we may obtain the following theorem.

THEOREM 4. For each Waraskiewicz spiral $W$, there exists a plane continuum $\hat{W}$ such that each nondegenerate subcontinuum of $\hat{W}$ can be mapped onto $W$.

THEOREM 5. If $X$ is a hereditarily indecomposable continuum and $\operatorname{dim} X>1$, then $X$ is not homogeneous.

ProOF. Let $x$ be a point of $X$, and let $[x, X]$ denote the space of all subcontinua of $X$ that contain $x$, with the topology generated by the Hausdorff metric. The continuum $[x, X]$ is homeomorphic to $[0,1]$. Let $\left\{X_{i}\right\}$ denote a countable dense set in $[x, X]$.

By Theorem 2, there exists a Waraskiewicz spiral $W$ such that no $X_{i}$ can be mapped onto $W$. By Theorem 4, there exists a continuum $\hat{W}$ in $I^{2}$ such that each map of $X_{i}$ into $\hat{W}$ is constant.

Since $\operatorname{dim} X>1$, there exists a weakly confluent map $f: X \rightarrow I^{2}$ of $X$ onto $I^{2}$. There exists a subcontinuum $Z$ of $X$ such that $f(Z)=\hat{W}$. If $X$ were homogeneous, we could assume $x \in Z$. For all $X_{i} \subset Z, f\left(X_{i}\right)$ is degenerate, and hence $f\left(X_{i}\right)=f(x)$. This implies the contradiction that $f(Z)=f(x)$. Hence $X$ is not homogeneous.

The preceding proof can be modified slightly to prove the following theorem. 
THEOREM 6. If $X$ is a hereditarily indecomposable continuum and $\operatorname{dim} X>1$, then $X$ has uncountably many orbits under the action of its homeomorphism group.

The conclusions of Theorems 5 and 6 remain true for all one-dimensional, hereditarily indecomposable continua that admit a weakly confluent map onto a higherdimensional continuum. In particular, the hereditarily indecomposable, one-dimensional continua constructed by Lewis $[\mathbf{5}]$ have uncountably many orbits under the action of their homeomorphism groups.

2. Aposyndetic decompositions. Burton Jones [4] has proved an aposyndetic decomposition theorem that is essential to the study of homogeneous continua.

THEOREM 7. If $X$ is a homogeneous, decomposable continua that is not aposyndetic, then $X$ admits a continuous decomposition into mutually homeomorphic, indecomposable, homogeneous continua such that the quotient space is an aposyndetic, homogeneous continuum.

The author [8] has shown that the elements of this aposyndetic decomposition must be cell-like continua. Now we observe the additional fact that if $\operatorname{dim} X=n$, then the elements of this decomposition are also $n$-dimensional.

A subcontinuum $Z$ of the continuum $X$ is said to be terminal if each subcontinuum $Y$ of $X$ that intersects $Z$ satisfies either $Y \subset Z$ or $Z \subset Y$. A decomposition of $X$ into continua is said to be terminal if each element of the decomposition is a terminal subcontinuum of $X$.

THEOREM 8. Let $\mathcal{G}$ be a continuous, terminal decomposition of the continuum $X$ into nondegenerate continua. If $\operatorname{dim} X \geq n$, then the dimension of some element of $\mathcal{G} \geq n$.

PROOF. Cover $X$ with open sets no one of which contains an element of $\mathcal{G}$. Let $O$ be a finite subcover. Some set $O$ of $O$ satisfies $\operatorname{dim} \bar{O} \geq n$. Some subcontinuum $K$ of $\bar{O}$ satisfies $\operatorname{dim} K \geq n$. Hence the dimension of the element of $\mathcal{G}$ containing $K \geq n$.

COROllary 9. If $X$ is a homogeneous, decomposable continuum that is not aposyndetic, then the elements of Jones's aposyndetic decomposition have the same dimension as $X$.

ProOF. Jones [4] has proved that his aposyndetic decomposition is terminal.

COROLlaRY 10. If $X$ is a continuum and $\operatorname{dim} X>1$, then there does not exist a continuous, terminal decomposition of $X$ into pseudo-arcs.

$\S \S 1$ and 2 blend together in the following fashion. No example of a homogeneous, indecomposable continuum $X$ with $\operatorname{dim} X>1$ is known. If one could prove Theorem 5 with only the hypothesis of "indecomposable" rather than "hereditarily indecomposable", then the results of this paper would imply that each homogeneous continuum $X$ with $\operatorname{dim} X>1$ is aposyndetic.

\section{REFERENCES}

1. R. D. Anderson and G. Choquet, A plane continuum no two of whose nondegenerate subcontinua are homeomorphic: an application of inverse limits, Proc. Amer. Math. Soc. 10 (1959), 347-353.

2. R. H. Bing, Higher-dimensional hereditarily indecomposable continua, Trans. Amer. Math. Soc. 21 (1951), 267-273. 
3. H. Cook, Treelikeness of hereditarily equivalent continua, Fund. Math. 68 (1970), 203-205.

4. F. Burton Jones, The aposymdetic decomposition of homogeneous continua, Topology Proc. 8 (1983), 51-54.

5. I. W. Lewis, Monotone maps of hereditarily indecomposable continua, Proc. Amer. Math. Soc. 75 (1979), 361-354.

6. T. Maćkowiak and E. D. Tymchatyn, Continuous mappings on continua. II, Dissertationes Math. 125 (1984), 1-62.

7. S. Mazurkiewicz, Sur l'existence des continues indécomposables, Fund. Math. 25 (1935), 327-328.

8. J. T. Rogers, Cell-like decompositions of homogeneous continua, Proc. Amer. Math. Soc. 87 (1983), 375-377.

9. __ Homogeneous, hereditarily indecomposable continua are tree-like, Houston J. Math. 8 (1982), 421-428.

10. R. L. Russo, Universal continua, Fund. Math. 105 (1979), 41-60.

11. Z. Waraskiewicz, Sur un probléme de M. H. Hahn, Fund. Math. 18 (1932), 118-137.

Department of Mathematics, Tulane University, New Orleans, Louisiana 70118 